\title{
Diabetes and kidney disease: insult added to injury
}

\author{
PETER H WINOCOUR
}

Diabetes and kidney disease are closely linked. In the UK and most Western countries, diabetes is considered the main cause of end stage renal failure. Although classical diabetic glomerulosclerosis manifesting as microalbuminuria with subsequent progressive loss of renal filtration function is most typically seen in type 1 diabetes, this remains an important contributor to advancing kidney disease in type 2 diabetes as well. However, the nature of renal disease in type 2 diabetes is much more heterogeneous and it may be more relevant to define many cases of kidney disease being with rather than due to type 2 diabetes.

Regardless of the precise nature of the relationship, it is abundantly clear that both diabetes and chronic kidney disease (CKD) are becoming more common, in large part a reflection of increasing longevity and obesity. ${ }^{1-3}$ The definition as to what constitutes CKD is based on estimates of glomerular filtration rate (eGFR) and, additionally, levels of albumin excretion. Both these measures have inherent variability and challenges as to their applicability to individual care, unless the limitations of these estimates and ratios are recognised. ${ }^{1}$ In addition, it has been argued that some inevitable physiological age-related changes in body mass and renal function can lead to what I have defined as the 'renopause', where inevitable changes in eGFR will lead to ostensibly healthy individuals (without diabetes) being labelled as having 'CKD' on primary care registers. ${ }^{3,4}$

The vast majority of the care of diabetes patients with renal disease takes place in primary care. The current DM Quality Outcome Framework has several metrics related to this, including annual measurements of eGFR and albuminuria and particular use of ACE inhibitors/angiotensin receptor blockade for hypertension, especially where there is microalbuminuria.

However, it is clear from the National Diabetes Audit that many fundamental care processes and surrogate outcome measures are infrequently attained in contemporary practice..$^{5}$ This pattern has been replicated in studies of specialist care in other parts of Europe and in Asia. ${ }^{6,7}$

Most guidelines in the UK and wider afield make broad recommendations about managing glycaemia and cardiovascular risk factors, and separately about assessing renal disease. ${ }^{8-10}$ However, with the exception of international consensus documents ${ }^{11,12}$ designed to be broadly applicable to all healthcare systems, there have to

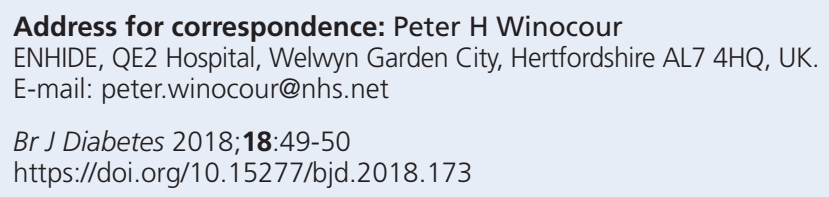

date been no specific diabetes guidelines to help manage these issues in the context of associated renal disease and, in particular, they have not taken account of type 1 and type 2 diabetes separately or the different stages of renal damage, mainly focusing on end stage renal disease. In addition, practical recommendations for clinicians have been evidently lacking.

$A B C D$ has worked closely with the Renal Association to rectify this. In addition to holding joint biannual educational meetings since 2012, we have worked collaboratively to develop clinical guidelines, which to date have all been published in the British Journal of Diabetes. All guidelines set out to offer evidence-based updated practical advice, linked to audit standards. In addition, areas of outstanding enquiry for future clinical research have been identified.

Our first published guideline was under the auspices of the Joint British Diabetes Societies (JBDS) and covered the practical aspects of coordinated care of diabetes patients on haemodialysis. ${ }^{13}$ This is scheduled for an updated publication in Diabetic Medicine later this year, but clearly needs wider utilisation in renal departments where the case for a diabetes or renal nurse clinical navigator is clear.

The management of lipid disorders in adults with diabetes and nephropathy (ie, albuminuric disease)/CKD was published early in 2017, emphasising the differences in lipid metabolism in type 1 and type 2 diabetes and the need to offer tailored therapy to higher risk younger patients whilst aiming to use high intensity statins (usually atorvastatin 40-80 mg) in most patients with established CKD. ${ }^{14}$ The restricted role of fibrates and their potential in microvascular protection was also commented on.

The joint guidelines on hypertension management and blockade of the renin-angiotensin-aldosterone system (RAAS) in diabetes and CKD was published at the end of $2017 .{ }^{15}$ It also continued the theme of separating the management of type 1 and type 2 diabetes and the different stages of renal disease, confirming the standard blood pressure targets of 130/80 mmHg when albuminuric and $140 / 90 \mathrm{mmHg}$ when normoalbuminuric. There were also clear messages regarding more proactive input in early nephropathy in younger adults (aged <40) with persistent albuminuria, and by contrast the need for a more conservative blood pressure target for many aged >75 years. In addition, the guidelines emphasised the potential role of RAAS blockade for retinal protection. The practical issues of dose-limiting hyperkalaemia and the current position of aldosterone antagonists was covered with a range of areas that need further research, including the potential for potassium binders given the challenge of RAAS blockade in diabetes patients with cardio-renal issues including cardiac failure, as well as in managing hypertension. 
This current issue of $B J D$ contains the most recent (and largest) output from the ABCD Renal Association Clinical Speciality Group - namely, managing hyperglycaemia in patients with diabetes and nephropathy-CKD. ${ }^{16}$ This addresses the issue of varying glycaemic targets and measurement of $\mathrm{HbA}_{1 \mathrm{c}}$ when anaemia supervenes. In addition, there are detailed discussions on the evidence for all classes of agents used in managing diabetes in CKD. The published guideline contains the summary recommendations, audit standards and suggestions for further research. The extended guidance is currently available on both the $A B C D$ and Renal Association websites.

This unique portfolio of guidelines will hopefully be of use in the challenges faced in both primary care and specialist diabetes healthcare settings, and we hope has resonance beyond these shores.

Going forward is the recognition that the management of diabetes and concomitant nephropathy-CKD is complex through frequent comorbidity. ${ }^{1}$ The primary focus may remain on monitoring progression of renal disease, appropriate management of hyperglycaemia and minimisation of the increased risk of acute kidney injury, at least while we have limited tools to change the natural history of renal disease beyond managing cardiovascular risk factors and hyperglycaemia. However, the potential for SGLT2 inhibitors as well as GLP1 analogues to directly benefit renal function and the continued interest in addressing the inflammatory and tissue hypoxic factors that progress CKD make this an exciting area of diabetes care.

The reality that $25-40 \%$ of those with diabetes have CKD as currently defined clearly requires a different approach from traditional models of care, not least given the evident unmet need and recognition that perhaps $80 \%$ of such patients currently receive standard care in overstretched primary care settings.

A new perspective on this comorbid disease complex is vital. If we only focus on the progression to end stage renal disease (important enough!), we fail to address the much wider issue of those with less advanced CKD whose enhanced vulnerability to hypoglycaemia, anaemia, feet, retinal, bone and cardiovascular complications and increased hospitalisation rates are often evident for years. ${ }^{1}$ The consequence will be an even greater burden and disproportionate economic costs to healthcare systems, ${ }^{17}$ which I fear will be unsustainable.

Conflict of interest Peter Winocour has received honoraria for delivering educational meetings and/or attending advisory boards for AstraZeneca, Eli Lilly, Novo Nordisk, Sanofi, MSD, Janssen and Vifor Pharmaceuticals.

\section{References}

1. Winocour PH. Diabetes and chronic kidney disease: an increasingly common multi-morbid disease in need of a paradigm shift in care. Diabet Med 2018;35:300-5. https://doi.org/10.1111/dme.13564
2. Hill CJ, Cardwell CR, Maxwell AP, et al. Obesity and kidney disease in type 1 and type 2 diabetes: an analysis of the National Diabetes Audit. Q J Med 2013;106:933-42. https://doi.org/10.1093/qjmed/hct123

3. Ellam T, Twohig H, Khwaja A. Chronic kidney disease in elderly people: disease or disease label. BMJ 2016;352:h6559. https://doi.org/10.1136/bmj.h6559.

4. Solini A, Penno G, Bonora E, et al. Age, renal dysfunction, cardiovascular disease, and antihyperglycemic treatment in type 2 diabetes mellitus: findings from the Renal Insufficiency and Cardiovascular Events Italian Multicenter Study. J Am Geriatr Soc 2013;61:1253-61. https://doi.org/10.1111/jgs.12381

5. Hill CJ, Cardwell CR, Patterson CC, et al. Chronic kidney disease and diabetes in the National Health Service: a cross-sectional survey of the UK National Diabetes Audit. Diabet Med 2014;31:448-54. https://doi.org/10.1111/dme.12312.

6. De Cosmo S, Viazzi F, Pacilli A, et al. Achievement of therapeutic targets in patients with diabetes and chronic kidney disease: insights from the Associazione Medici Diabetologi Annals initiative. Nephrol Dial Transplant 2015;30:1526-33. https://doi.org/10.1093/ndt/gfv101

7. Luk AO, Li X, Zhang Y, et al. Quality of care in patients with diabetic kidney disease in Asia: The Joint Asia Diabetes Evaluation (JADE) Registry. Diabet Med 2016;33:1230-9. https://doi.org/10.1111/dme.13014

8. American Diabetes Association. Standards of medical care in diabetes2018. J Clin Appl Res Educ 2018;41(Suppl 1):S1-156.

9. Lipscombe L, Booth G, Butalia S, et al. Pharmacological glycaemic management of type 2 diabetes in adults. 2018 Clinical Practice Guidelines. Can J Diabetes 2018:42:588-103. https://doi.org/10.1016/j.jcjd.2017.10.034

10. National Institute for Health and Care Excellence. Type 2 diabetes in adults: management. [NG28]. 2015. Available at www.nice.org.uk/guidance/ng28 (accessed June 2018).

11. KDOQI (Kidney Disease Outcomes Quality Initiative) clinical practice guideline for diabetes and CKD: 2012 update. Am J Kidney Dis 2012; 60:850-86. https://doi.org/10.1053/j.ajkd.2012.07.005

12. Bilo H, Coentrao L, Couchard C, et al. Guideline Development Group. Clinical practice guideline on management of patients with diabetes and chronic kidney disease stage $3 \mathrm{~b}$ or higher (eGFR $<45 \mathrm{~mL} / \mathrm{min}$ ). Nephrol Dial Transplant 2015;30(Suppl 2):ii1-142. https://doi.org/10.1093/ndt/gfv100

13. Frankel A, Kazempour-Ardebili S, Bedi R, et al. Management of adults with diabetes on the haemodialysis unit: summary of new guidance from the Joint British Diabetes Societies (JBDS) and the Renal Association. $\mathrm{Br}$ J Diabetes 2016;16:69-77. https://doi.org/10.15277/bjd.2016.073

14. Mark PB, Winocour P, Day C. Management of lipids in adults with diabetes mellitus and nephropathy and/or chronic kidney disease: summary of joint guidance from the Association of British Clinical Diabetologists (ABCD) and the Renal Association (RA). Br J Diabetes 2017;17:64-72. https://doi.org/10.15277/bjd.2017.125

15. Dasgupta I, Banerjee D, Chowdhury TA, et al. Association of British Clinical Diabetologists (ABCD) and Renal Association clinical guidelines: hypertension management and renin-angiotensin-aldosterone system blockade in patients with diabetes, nephropathy and/or chronic kidney disease. Summary of recommendations. Br J Diabetes 2017;17:160-4.

16. Winocour P, Bain S, Chowdhury TA, et al. Managing hyperglycaemia in patients with diabetes and diabetic nephropathy-chronic kidney disease. Br J Diabetes 2018;18:78-89. https://doi.org/10.15277/bjd.2018.172

17. Vupputurri S, Kimes TM, Calloway MO, et al. The economic burden of progressive kidney disease among patients with type 2 diabetes. J Diabetes Complications 2014;28:10-16.

https://doi.org/0.1016/j.jdiacomp.2013.09.014 